\title{
Diagnostic Techniques of Oral Candidosis: A Review
}

\author{
Shubhangi Khandekar ${ }^{1}$, Alka Dive ${ }^{2}$, Neha Upadhyaya ${ }^{3,}$ \\ Rakesh Kumar Mishra ${ }^{4}$, Shalini Gupta ${ }^{5}$, Rohit Moharil ${ }^{6}$ \\ 1,2,3,6(Department of Oral \& Maxillofacial Pathology, V.S.P.M.D.C.R.C,Nagpur,India) \\ 4(Department of Ear,Nose \& Throat,N.K.P.S.I.M.S,Nagpur,India) \\ 5(Department of Oral \& Maxillofacial Pathology,C.S.M.M.U,Lucknow,India)
}

\begin{abstract}
Candidosis is said to be the most opportunistic infection in the world. This can result from many different conditions like irrelevant antibiotic treatment, dentures, corticosteroid therapy, cancer chemotherapy, diabetes mellitus, HIV infection, hypoparathyroidism, infancy, multiple myeloma, primary T-lymphocyte deficiency, xerostomia. This review attempt at spreading awareness among clinicians regarding isolation \& accurate diagnosis of yeast in their practice to save \& serve mankind in a better way.
\end{abstract}

Keywords: Oral Candidosis, Candida albicans ,Laboratory Diagnosis

\section{Introduction:}

The outcome of an overgrowth of Candida albicans causes oral candidosis . The majority of infections are due to Candida albicans (C. albicans) although other species such as C. glabrata, C. tropicalis, C. Krusei and C. parapsilosis are increasingly isolated. In fact, C.albicans may be a component of normal oral microflora, with as many as $30 \%$ to $50 \%$ of people simply carrying the organism in their mouths without clinical evidence of infection. The frequency of invasive fungal infections has increased over the last decade ${ }^{1,2}$. A correct diagnosis provides the specific treatment of a fungal infection and may prove life saving or stave off the complications produce there in $^{3}$.

\section{Isolation of Yeast from Oral Samples:}

The following are the guidelines for specimen collection.

1. The specimen should be collected from an active lesion; old 'burned out' lesions often do not contain viable organisms.

2. Collect the specimen under aseptic conditions.

3. Collect sufficient specimen.

4. Use sterile collection devices and containers

5. Label the specimen appropriately; all clinical specimens should be considered as potential biohazards and should be handled with care using universal precautions ${ }^{4}$.

SMEAR: Smears taken from the lesional tissue can be seen directly in the microscope. This is a simple and valuable method in the diagnosis of candidosis .

SWAB: A sterile cotton tipped swab is rubbed over the lesional tissue.Swab should be conveyed to the laboratory as quickly as possible. C.albicans can survive atleast $24 \mathrm{hrs}$ on moist swab without loss of viability. Immersion in buffered charcoal or in an enrichment medium can increase the isolation sensitivity.

IMPRINT CULTURE TECHNIQUE : Sterile plastic foam pads are dipped in Sabouraud's broth \& placed on the surface under study for about 60 seconds. The pad is then pressed firmly on to Sabouraud's plate \& send to laboratory. It is a sensitive \& reliable method for oral sampling. Disadvantages of imprint culture is that a number of areas may have to be sampled, the appropriate sites for sampling is subjective, there can be accidental risk of inhalation of the foam pad by the patient and lastly,the need to store sterile foam pads or culture media in the clinic.

IMPRESSION CULTURE TECHNIQUE : This method is totally a research tool \& is useful in quantifying the relative distribution of yeasts on oral surfaces such as the teeth,63odeled63 \& palate. Maxillary \& Mandibular alginate impressions are taken \& transported to laboratory, casted in $6 \%$ fortified agar with incorporated Sabouraud's dextrose broth, incubated \& CFU(colony forming unit) of yeast estimated.

SALIVARY CULTURE TECHNIQUE: This technique is a sensitive \& as accurate as an imprint culture .But the method involves considerable chair time, depending on the salivary flow rate of the patients. This simple technique involves requesting the patient to expectorate $2 \mathrm{ml}$ of mixed unstimulated saliva into a sterile, 
universal container which is then vibrated for 30 second on a bench vibrator for optimal disaggregation. The number of Candida expressed as CFU/ml of saliva is estimated by counting the resultant growth on sabouraud's agar using either the spiral plating or surface viable counting technique.

ORAL RINSE TECHNIQUE: This method consists of requesting the subjects to rinse the mouth for 60 seconds, with $10 \mathrm{ml}$ of phosphate-buffered saline supplied in a universal container.The patient then returns oral rinse to the universal container, which is sent to the laboratory. In the laboratory the oral rinse is concentrated by spinning for 10 minutes \& resuspending the deposits in $1 \mathrm{ml}$ of sterile PBS(phosphate-buffered saline).The concentrated oral rinse is then 64odeled64ed on appropriate media to assess $\mathrm{CFU} / \mathrm{ml}$ of rinse sample using a spiral plater or Gallenkamp colony counter ${ }^{5}$.

CANDIDA ISOLATION IN THE CLINIC :There are atleast 2 commercially produced ,rapid systems, which may be suitable for dentists to assess oral Candida in their practices, namely, Microstix-Candida \& OricultN.These tests are 64odeled on the principle of the dip slide technique. The rapid commercial systems for diagnosing oral candidosis are useful for screening patients in the clinical setting,particularly when microbiology laboratory is not within easy access. The Microstix-candida (MC) system consists of a plastic strip to which is affixed a dry culture area $(10 \mathrm{~mm} \times 10 \mathrm{~mm})$ of modified Nickerson medium (Nickerson, 1953)and a plastic pouch for incubation. The Yeast-I dent system is based on the use of chromogenic substances to measure enzyme activities. Oricult-N dip slide technique is similar to, but of higher sensitivity than M-C system. Yeast-I dent system is based on the use of chromogenic substances to measure enzyme activities ${ }^{6}$.

\section{Culture media:}

\section{Identification Of Candida Species :}

Most useful \& popular primary culture media is Peptone - glucose (dextrose) or peptone - maltose agar .It was first described in 1896 by Sabouraud \& hence known as "Sabouraud's agar ", which shows Candida colonies as cream coloured smooth or rough, shiny or dull,convex in appearance.Candida albicans can also be formed on blood agar,where they appear as moist, opaque,whitish colonies characteristic of yeast.Candida albicans on a Dalmau plate appears as clusters of round blastoconidia are present at some septae,with thickwalled terminal chlamydospores (characteristic of $C$. albicans) are seen .Pagno - Levin Media allows various yeast species to be distinguished through visual differences in coloration eliciting degrees of pink ,blue and orange colouration.. The medium is superior in detecting multiple yeast species in a single sample. Chrom Agar is another media detecting multiple yeast species in a single sample CHROM agar can differentiate C.albicans as green colonies, C. krusei as pink colonies and C.tropicalis as blue colonies.

\section{Morphological and Physiological Tests:}

Identification of candida species can also be done by several morphological and physiological tests.Direct microscopy of the Gram stained smears show characteristic rounded, or oval budding cells of yeast blastospores (yeast form) with or without the hyphal phase, which can be distinguished readily from bacteria by their greater size $(3-6 \mu \mathrm{m})$, budding, oval shape \& pseudohyphae. Water mount /wet film of yeast suspension is made on a glass slide, using sterile distilled water .This emulsion is mounted with a coverslip \& examined under X 100 or X 400 magnification. $\mathrm{KOH}$ acts as cleaning agent(lyses the background of epithelial cells), eliminating most debris \& intensifying the contrast of fungal structures,can be used for direct examination of mucosal scrapings ${ }^{5}$.A germ tube is a filamentous, cylindrical outgrowth from the yeast cell with no constriction present at base is a rapid screening procedure for differentiating $\mathrm{C}$. albicans from other species.Chlamydospore is a thick walled, non deciduous,asexual spore formed by rounding off cell or cells. They are thought to be dormant growth forms produced under conditions of nutrient depletion.This Property is peculiar to C. albicans \& to very rare isolates of C. tropicalis \& C. stellatoidea .Physiological test include the Carbon \& Nitrogen assimilation test and Carbohydrate fermentation test .C. albicans ferments glucose and maltose more rapidly than other sugars ${ }^{5}$.

Cellular and molecular mechanisms utilized in identification of various candida species : Oral Candida organisms have a manner in which they adhere to the oral mucosa as well as to non-oral surfaces (such as the denture) via the use of biofilms. Candida continues to exist intraorally because they are able to form biofilms that allow them to grow and flourish. It has been noted that it is the structure of the cell wall of Candida that displays properties that promote the formation of the biofilms, thereby allowing it to infiltrate the tissue and ultimately inhabit various organs in the body, including the oral mucosa . Candida albicans is able to utilize special mechanisms that allow it to be able to switch back and forth from one form to another (from budding yeast form to pseudohyphal to hyphal forms). For this reason, the formation of Candida biofilms creates serious clinical injurious effects because of their increased resistance to antifungal therapy; the cells within 
biofilms also carry the capability to resist immune defenses of the host. This propensity to adhere to host cells of the epithelium is a virulence factor of species of oral Candida.

Quorum- Sensing : Additionally, it has been found that Candida species and bacteria communicate with one another via a mechanism known as "quorum- sensing.".Candida and bacteria utilize signaling mechanisms where they have a direct effect on each other. C. albicans employs two major quorum sensing molecules known as tyrosol and farnesol. Tyrosol has the ability to stimulate the formation of hyphae in C. albicans while farnesol inhibits the shift from the yeast form to the hyphal form and regulates the morphology of the cell .Quorum- sensing is a phenomenon where the accrual of signaling molecules enables a single cell to sense the number of bacteria/fungi that are present,this allows bacteria/fungi to manage and organize the way they behave so that they are able to respond quickly to their changing environments in order to sustain themselves ${ }^{7,8}$

\section{Rapid Commercial Systems For Identification Of Clinically Important Yeasts :}

\begin{tabular}{|l|l|}
\hline - Integral diagnostic systems & - Instrumental systems \\
- API 20 C & - Abbott MS-2 \\
- Auxodisk & - Autobac 1 \\
- Iatron Candida check & - Auto Microbic System \\
- Micro-drop & \\
- Minitek(BBL) & \\
- Mycotube(Roche) & \\
- Uni-Yeast-Tek & \\
- Randolph Multitest Mycology Plate & \\
- Yeast Ident & \\
\hline
\end{tabular}

\section{STRAIN DIFFERENTIATION WITHIN CANDIDA SPECIES :}

SEROTYPING: C.albicans strains can be serotyped as Serotype A \& Serotype B, based on the antigenic characteristics of their cell walls. This is done by simple slide agglutination.A more discriminatory serotyping is yet to be developed due to the advent of monoclonal antibody technique.

BIOTYPING: A combination of APIZYM system,API 20C system \& a plate test for resistance to boric acid has been developed for biotyping C.albicans.It has provided 234 possible biotypes. This system should help overcome irregularities in media preparation \& interpretation of data from different centres,thus generating universally comparable data.

RESISTOGRAM TYPING: This method is based on the differences in resistance of the candida isolates to six selected organic \& inorganic chemicals incorporated in agar media .

\section{OTHER TYPING METHODS :}

- Biotyping based on yeast killer factors

- Immunoblotting

- Colony morphotyping

- Electrophoretic karyotyping

- DNA restriction fragments length polymorphisms. ${ }^{5}$

\section{Polymerase Chain Reaction (Pcr) Fingerprinting:}

Polymerase Chain Reaction (PCR) fingerprinting has been utilized to differentiate between various strains of C. Albicans and C. dubliniensis. This is a challenging problem because of the high degree of similarity in the phenotype between C. dubliniensis and C. Albicans ${ }^{9}$. Multiplex PCR has been used to identify various Candida species, particularly C. Albicans, C. glabrata, and C. tropicalis in a single sample ${ }^{20}$. Furthermore, PCR has been successfully used to identify C. dubliniensis in gingival fluid of healthy individuals with periodontal disease. Tests were identified using conventional sampling methods as well as specific PCR assays. Results showed that $\mathrm{C}$. dubliensis was isolated and identified in subgingival fluid of periodontal pockets of persons with healthy immune systems. Realtime PCR have demonstrated that Candida biofilms can exert resistance to many commonly employed antifungals in the clinical setting. Real-time PCR chemistries allow for the detection of PCR amplification during the early phases of the reaction, measuring the kinetics during the early phases provides an advantage over the traditional PCR where agarose gels are used for the detection of PCR amplification at the final endpoint phase of the reaction . C. Albicans genes have also been identified that regulate the communication between oral epithelial cells involved in oropharyngeal candidiasis and vascular 
endothelial cells. These genes increase their interaction as they come into contact with epithelial or endothelial cells and as they endure the stresses that they likely encounter as they grow in the oropharynx and in the blood .

\section{Recombinant Dna:}

Use of recombinant antigens for the diagnosis of invasive candidiasis has been recently studied . The detection of antibodies directed against antigens which are expressed on the C. albicans germ tube surface by indirect immunofluorescence has been shown to be valuable for diagnosis. Among the Candida enzymes detected in sera of patients who have systemic candidiasis, enolase has been found to be highly diagnostic. Advances in molecular biological techniques have also allowed for the completion of the Candida albicans genome sequence. Overall, these molecular biology techniques have allowed the production of recombinant antigens which are useful for the discovery and identification of antibodies against them. It has been displayed that the detection of antibodies against purified and well-defined recombinant antigens allows for the diagnosis of Candidal organisms which are invasive in nature ${ }^{10}$.

\section{Immunological Methods:}

Denture stomatitis is a very real and common problem within the dental setting and a significant portion of this has been reviewed here with regards to immunologic aspects. It was noted in another investigation, that $C$. Albicans is the predominant fungal species isolated from those who wear dentures and that C. dubliniensis was isolated from the underlying mucosa but not from the prosthetic surface. C.glabrata has been found to be an opportunistic pathogen in individuals who use long term immunosuppressants and broadspectrum antibiotics-especially in the elderly.This may be due to several factors, including decreased salivary gland hypofunction as well as $\mathrm{pH}$ changes noted in the elderly. The $\mathrm{pH}$ of the oral mucosa in the elderly appears to be more acidic than the oral $\mathrm{pH}$ of younger people ${ }^{11}$.

\section{Detection Of Candida In Biopsy Specimens :}

Because candida spp. are poorly stained by routinely used H\&E stain ,they may be overlooked when a tissue section is examined. However, periodic acid -Schiff (PAS) \& Gridley's or Gomori's methenamine silver (GMS) stains are ideal for delineating the fungal elements. Calco Fluor White is a water soluble colourless dye that selectively and permanently binds to $\beta$-1,4-glycosidic linked polysaccharides (cellulose).By exposing CFW to UV light, its transisomer will fluoresce apple green /light blue. Other specialized stains include the Wright's stain, Periodic acid - basic fuschin - light green, Acridine orange, Fluorescein- conjugated lectins, Woolfast pink RL methylene blue

\section{Treatment :}

Treatment can be accomplished with the use of appropriate antifungal medications and routine disinfection of removable intraoral prostheses. Both topical and systemic medications have been used. The development of new specific antifungal agents such as nystatin has been beneficial in the treatment of candidosis. Other drugs of value are Chlotrimazoles, Amphotericin-B and Iconazole.It has been found that occasional cases of candidosis have remained refractory to treatment by Nystatin .They have frequently been associated with one of the endocrinopathies just described in connection with immunologic abnormalities ${ }^{1}$. Recent developments in the treatment of oral candidiasis include the use of probiotic bacteria which are known to reduce the growth of pathogenic microbes. A recent study used cheese containing a mixture of probiotics such as Lactobacillus rhamnosus GG, Lactobacillus rhamnosusLC705, Propionibacterium freudenreichii and shermanii JS. The use of probiotic cheese revealed the reduction of the prevalence of oral Candida in the elderly and its use also allowed for the reduction of the prevalence of hyposalivation ${ }^{12}$.

\section{Conclusion:}

It is evident that no single method is sensitive enough for universal recommendation for isolation of yeast. Finally, a plea should be made to standardize the sampling methods used in detecting oral candidosis in order to generate universally comparable data ${ }^{5}$. Various cellular and molecular mechanisms have been employed to help elucidate the interactions between Candida organisms and their environment ${ }^{11}$. DNA finger printing has higher discrimination \& superior sensitivity.Also, the wide availability of basic equipment for DNA recombinant technology, DNA fingerprinting of Candida may well be the typing system of the future ,supplanting the techniques used currently. Polymerase chain reaction analyses, Candida biofilm communication, and recombinant antigen techniques have been found to facilitate explanations of the endurance and continued existence of oral candidiasis, specifically C. albican ${ }^{l 1}$. Although considerable progress has been made in the understanding of oral candida infection and their treatment much remains to be done. 


\section{References:}

[1] Rajendran R., Shivapathasundharam B. Shafer"es text book of oral pathology, $5^{\text {th }}$ ed. India: elsevier; 2005.

[2] Parihar.S,Oral Candidiasis- A Review, WebmedCentral

[3] Jagadish Chander. Textbook of Medical Mycology 2nd edition Mehta Publishers 2002; 40-53.

[4] Epstein J B, Pearsall N N, Trulove E L.Oralcandidosis: effects of antifungal therapy upon clinical signs and symptoms, salivary antibody and mucosal adherence of C.albicans. Oral Surg Oral Med Oral Pathol 1981; 51:32-38.

[5] Samaranayake LP, Yaacob H. Classification of oral Candidoses: In Oralcandidoses. London:Butterworth 1990. Pg No 124-132

[6] Olsen I and Stenderup A. Clinical - mycologic diagnosis of oral yeast infections. Acta Odontol Scand.1990; 48:18

[7] ten Cate JM, Klis FM, Pereira-Cenci T, Crielaard W, de Groot PWJ. Molecular and cellular mechanisms that lead to Candida biofilm formation. J Dent Res 2009; 88: 105-15.

[8] Hornby JM, Jensen EC, Lisec AD, et al. Quorum sensing in the dimorphic fungus Candida albicans is mediated by farnesol. Appl Environ Microbiol 2001; 67(7): 2982-92.

[9] Neppelenbroek KH, Campanha NH, Spolidorio DM, Spolidorio LC, Seo RS, Pavarina AC. Molecular fingerprinting methods for the discrimination between Candida Albicans and Candida dubliensis. Oral Dis 2006; 12(3): 242-53

[10] Liquori G, Di Onofrio V, Luvariello A, et al. Oral Candidiasis: a comparison between conventional methods and multiplex polymerase chain reaction for species identification. Oral Microbiol Immunol 2009; 24(1): 76-8.

[11] Celeste M. Abraham, Advances and Emerging Techniques in the Identification, Diagnosis and Treatment of Oral Candidiasis, The Open Pathology Journal, 2011, 5, 8-12.

[12] Hatakka K, Ahola AJ, Yli-Knuuttila $\mathrm{H}$, et al. Probiotics reduce the prevalence of oral Candida in the elderly--a randomized controlled trial. J Dent Res 2007; 86; 125-30. 